\title{
Derecho y literatura Law and Literature
}

\author{
María Jimena Sáenz ${ }^{*}$ \\ CONICET-UNLP \\ mjimenasaenz@hotmail.com
}

Recibido / received: 31/05/2018

Aceptado / accepted: 31/01/2019

DOI: https://doi.org/10.20318/eunomia.2019.4706

\section{Resumen}

Si bien el diálogo entre el derecho y la literatura puede parecer una pareja por lo menos extraña dentro de la explosión de estudios interdisciplinarios que tuvo lugar avanzada la segunda mitad del siglo xx, ambas disciplinas parecieron encontrarse en un marco institucional rastreable hacia la década de 1970 en la academia jurídica angloamericana. Ese marco institucional llevó el nombre de "movimiento derecho y literatura" y tuvo como objeto reflexionar sobre las relaciones entre ambos términos. Este trabajo presenta un repaso de la trayectoria del movimiento "derecho y literatura", sus distintas variantes, autores e instituciones clave. Ofrece una periodización del trabajo realizado a lo largo de los últimos casi cuarenta años hasta desembocar en el campo incipiente de la "literatura y los derechos humanos", y revisa la expansión del movimiento a través de las fronteras nacionales.

\section{Palabras clave}

Movimiento derecho y literatura, narración, interpretación, estudios culturales, literatura y derechos humanos.

\begin{abstract}
The relationship between law and literature is the object of study of the "law and literature movement", an interdisciplinary enterprise that emerged in the 70s in the context of Anglophone academy. This paper offers a review of its origins, key authors, contexts and institutions, and presents a periodization of the work done in more than three decades, its migrations to the field of literary studies and the expansion across different legal cultures.
\end{abstract}

\section{Keywords}

Law and literature movement, narration, interpretation, Cultural Studies, literature and human rights.

SUMARIO. 1. Introducción: las relaciones entre derecho y literatura. 2. Las etapas del movimiento: preocupaciones, instituciones, autores, contextos. 3. La expansión de las fronteras. 4. La migración de los estudios literarios. 5. A modo de cierre.

\footnotetext{
*Agradezco a los árbitros anónimos de la revista por sus observaciones y sugerencias que ayudaron a mejorar este trabajo..
} 


\section{Introducción: las relaciones entre el derecho y la literatura}

Las relaciones entre el derecho y la literatura, a pesar de la extrañeza de la pareja, tienen una historia larga y ampliamente narrada. Desde la época clásica, la literatura se ha involucrado con los personajes, temas y problemas del derecho; varios de los grandes escritores han sido abogados, trabajado en tribunales, en estudios de abogados, o ejercieron la función pública; ${ }^{1} \mathrm{y}$, por otro lado, hasta la segunda mitad del siglo XIX, "el derecho no constituía una rama de estudio autónoma, sino que la formación jurídica era una parte central de los estudios en humanidades" (Amaya, 2013). Esa historia fue retomada e institucionalizada durante la segunda mitad del siglo xx en el ámbito angloamericano bajo la denominación de "movimiento derecho y literatura".

El hito fundacional del movimiento suele situarse en 1973, con la publicación de The Legal Imaginationde J.B. White ${ }^{2}$; desde ese momento, uno de los impulsos tan tempranos como persistentes que caracterizaron al movimiento ha sido el intento de generar taxonomías que expliquen y justifiquen las relaciones entre el derecho y la literatura, y sobre todo, que autoricen y cubran bajo un manto común a la diversidad de trabajos, agendas de discusión y autores que se agrupaban bajo ese rótulo. Una de las más perdurables es la propuesta por Weisberg en 1989. Allí distinguía dos líneas de investigación: el "derecho en la literatura" y el "derecho como literatura". La primera involucraba el análisis de "la aparición de temáticas jurídicas o la representación de actores o procesos legales en la ficción o el drama" y tiene su antecedente más remoto en la lista de "novelas legales" de Wigmore publicada por primera vez en 1908. La empresa del "derecho como literatura", en cambio, "involucra el análisis gramatical de textos legales como la legislación, constituciones, sentencias judiciales y ciertos tratados académicos clásicos como si fueran obras literarias" (1989: 1) y puede considerarse que tiene su antecedente más remoto en el clásico trabajo de B. Cardozo de 1925. Si en los inicios del movimiento esta taxonomía pudo resultar de utilidad, el desarrollo posterior empezó a cruzarlas volviéndolas indistinguibles; y por otro lado, empezó a funcionar como una especie de carrera lineal del movimiento que iba desde los tempranos trabajos que leían obras literarias en búsqueda de nuevos pensamientos sobre el derecho, hacia el trabajo deseable "derecho como literatura"-que asumía la asimilación del derecho a la literatura e importaba teoría literaria para leer textos legales. ${ }^{3}$

Otra forma de distinguir líneas en el movimiento es comenzar por la pregunta sobre cómo están configuradas las relaciones entre el derecho y la literatura: si esa relación es una de asimilación y colonización del "otro" disciplinar (Balkin, 1996), o si se parte de la diferencia y se pone a funcionar un diálogo entre ambos puntos que no trae grandes marcos analíticos, sino pequeños encuentros que pueden iluminar aspectos sobre ambos interlocutores (Sáenz, 2017; Anker y Meyler, 2017: 8-10). Dos autores clásicos del movimiento, en reconstrucciones situadas en dos momentos diferentes, sugirieron esta reorientación. Por un lado, K. Yoshino (2006) planteó que

\footnotetext{
${ }^{1}$ Basta citar algunos ejemplos que comienzan con la figura de Goethe, gran parte de los escritores fundadores de las distintas tradiciones literarias alemanas (T. Storm, Hoffman, i.a.); al igual que Balzac y Flaubert en el caso francés; Dickens en el período victoriano inglés; Stevenson en Escocia. Este punto quizás explique la presencia abrumadora de la literatura victoriana y la novela realista en los trabajos en derecho y literatura, comenzando por la lista clásica de "novelas legales" de Wigmore. Ello pues, por un lado, el realismo decimonónico fue el terreno en el que se dirimió la "autonomía" de la literatura frente a otras esferas (Bourdieu 1995). Y por otra parte, esa preferencia del movimiento hacia los escritores del siglo XIX busca reafirmar que la articulación "derecho y literatura" ya estaba allí desde aquel momento. 2 Suelen citarse como antecedentes dos textos de principios de siglo: la lista varias veces revisada y ampliada de Wigmore (1908); y "Law and Literature" de B. Cardozo (1925).

3 Un ejemplo de este tipo de lecturas es el recuento completo del movimiento de Binder y Weisberg (2000).
} 
detrás de la clasificación del derecho en la literatura y el derecho como literatura estaban funcionando dos ideas de la literatura en cuestión: una "particularizante", que la ceñía a las obras de ficción, y otra "generalizante", que diluía lo literario en toda clase de discursos y producciones de registro escrito. En ese marco, señalaba la mayor comprensividad de la nueva clasificación, que tenía la capacidad de albergar líneas de trabajo como la regulación legal de la literatura, así como trabajos que leen obras literarias no solamente en sentido representacional sino también con cierta sensibilidad hacia la forma literaria (i.e. Nussbaum 1997 y 2006). Por su parte, en la reconstrucción temprana del movimiento de B. Thomas (1991), se señalaba que gran parte de la producción en "derecho y literatura" enfatiza demasiado en las semejanzas, y se sugería en cambio, que "son precisamente las diferencias lo que vuelve productivo relacionar la literatura y los documentos legales, no para fundirlos, sino para interrogarlos mutuamente (...) diluir el uno en el otro nos da tanto una visión distorsionada del derecho como de la literatura" (1991: 532-535).

Finalmente, el énfasis en la semejanza o la diferencia permitirá comprender estadios más avanzados del movimiento y conectar su trabajo con una discusión central en los últimos años en el campo literario: cómo defender a las humanidades en un contexto de precarización institucional y de crisis del valor.

En lo que sigue, el trabajo se organiza del siguiente modo. La sección 2 aprovecha la nueva orientación sugerida en los estudios en "derecho y literatura" para plantear una periodización del trabajo del movimiento, repasando sus autores, temas centrales y sus contextos de emergencia. Luego (sección 3), se esboza la expansión del derecho y la literatura más allá de las fronteras de origen en USA y su migración desde las escuelas de derecho que lo albergaban mayoritariamente hacia los estudios literarios. Finalmente, se ofrecen algunas reflexiones de cierre.

\section{Las etapas del movimiento: preocupaciones, instituciones, autores, contextos.}

La nueva taxonomía de la producción del movimiento que esquematiza dos polos ideales hacia los que se orientan los trabajos -la asimilación y la diferencia- ofrece una clave para seguir el desarrollo histórico del "derecho y la literatura" que puede leerse como una oscilación entre ambos proyectos que conviven todo a lo largo. Asimismo, permite distinguir dos tipos de miradas, dos vocabularios para pensar la relación, dos tipos de materiales "literarios" diferentes a los que se recurre, y recuperar las formas en que se canalizó una insatisfacción generalizada con el estado de la educación e investigación jurídica durante el último tercio del siglo XX.

Esa insatisfacción en el campo jurídico, que se vincula en gran parte de la bibliografía del movimiento a una "estrechez" de miras y preocupaciones, puede especificarse de dos modos diferentes y busca también una resolución literaria de dos maneras también diferenciables. Una de ellas puede caracterizarse como una ansiedad por dotar de más autoridad académica al derecho en un momento en que era percibido como una disciplina "profesional", que se resuelve con la importación de Teoría literaria; la otra, a partir de la necesidad no de elevar aún más al derecho, sino de hacerlo descender de los marcos abstractos y prolijos a gran escala hacia lo que Nussbaum describe como "la confusa vida de los seres humanos", donde se reúne con la literatura, en particular la novela, dando forma a un derecho "a escala humana". Ambas salidas del derecho hacia la literatura pueden distinguirse por su ubicación en dos polos: uno que piensa la relación a partir de la asimilación y recurre a la Teoría; otro que enfatiza no la semejanza, sino la diferencia literaria, recurriendo a la literatura narrativa o más específicamente a la novela para ponerla en diálogo con el derecho. 
La periodización más atractiva del movimiento segmenta: una primera etapa "humanista" desarrollada entre 1970 y 1980; una segunda etapa "hermenéutica" dominante entre 1980-1990 que aglutina trabajos centrados en la problemática común de la "interpretación", con fuerte impronta teórica que deja atrás a las obras literarias; un tercer momento "narrativo" durante los noventas, que vuelve hacia la literatura en su aspecto "narrativo" y reacciona frente a los excesos de hermetismo de la teoría; y una cuarta etapa de "estudios culturales" que vuelve a aplanar la diferencia literaria para situarla en el terreno más general de la cultura (Baron, 1999 y Peters, 2005). El esquema general que siguen las etapas de la producción del movimiento puede leerse como un movimiento pendular entre el énfasis en la diferencia literaria (etapas 1 y 3 ) y la asimilación (etapas 2 y 4); así como una oscilación entre dos tipos de miradas, dos tipos de materiales literarios a los que se recurre y dos vocabularios para pensar la relación. El cuadro siguiente presenta un esquema de esos cambios y oscilaciones.

\begin{tabular}{|l|c|c|c|c|c|c|c|}
\hline & \multicolumn{2}{|c|}{} & \multicolumn{2}{|c|}{ Mirada } & \multicolumn{2}{c|}{$\begin{array}{c}\text { Tipo y vocabulario } \\
\text { de la relación }\end{array}$} & \multicolumn{2}{c|}{ Material } \\
\hline \multicolumn{1}{|c|}{ Momentos } & Período & $\begin{array}{c}\text { Hacia } \\
\text { arriba }\end{array}$ & $\begin{array}{l}\text { Hacia } \\
\text { abajo }\end{array}$ & Asimilación & Diálogo & “Teoría” & novela \\
\hline 1. Humanista & $70 \mathrm{~s}$ & & $\mathrm{X}$ & & $\mathrm{X}$ & & $\mathrm{X}$ \\
\hline 2.Hermenéutico & $80 \mathrm{~s}$ & $\mathrm{X}$ & & $\mathrm{X}$ & & $\mathrm{X}$ & \\
\hline 3. Narrativo & $90 \mathrm{~s}$ & & $\mathrm{X}$ & & $\mathrm{X}$ & & $\mathrm{X}$ \\
\hline $\begin{array}{l}\text { 4.EstudiosCulturales } \\
\text { del derecho }\end{array}$ & $\begin{array}{c}\text { Post- } \\
2000\end{array}$ & $\mathrm{X}$ & & $\mathrm{X}$ & & $\mathrm{X}$ & \\
\hline
\end{tabular}

Brevemente, el primer período "humanista" está recortado por el tramo temporal delineado de manera amplia por dos de los hitos fundacionales del movimiento: la publicación en 1973 de The Legal Imaginationde White y la aparición de dos publicaciones dedicadas al área como son el Yale Journal of Law\&theHumanities (1988-89) y la revista Law and Literature de la Cardozo LawSchool (1988). Tomando de manera estricta la década de los 70 como el momento de mayor presencia del "humanismo", podría señalarse como punto de corte la fundación del Law and HumanitiesInstitute en 1978, que corona su trayecto y comienza también su declive ${ }^{4}$. Ese primer momento estuvo marcado por una preocupación reactiva hacia el rumbo que había tomado la educación y la práctica jurídica dominada por "una tendencia a aproximaciones neutrales valorativamente y tecnocráticas" en la que el vocabulario jurídico ofrecía una barrera a los juicios valorativos y encubría la responsabilidad por ese juicio (Sarat, 1998: 402). En palabras de Nussbaum, el movimiento estaba inspirado y reaccionaba contra la "estrechez de la educación e investigación jurídica, preocupadas exclusivamente por problemas doctrinales y que habían hecho poco por investigar problemas más amplios sobre los seres humanos y sus actividades en el mundo y en la sociedad a la que el derecho se dirigía" (Nussbaum y LaCroix, 2013: 9). En particular, Nussbaum ilumina el rival específico del movimiento: la visión económica estrecha que propugnaba el "movimiento derecho y economía" como ciencia, frente a la cual y de la cual debía nutrirse y medirse el derecho. La literatura, y en particular la novela, era para los humanistas una vía para situar a los valores en el centro de la discusión jurídica y devolverle así al derecho la dimensión humana que estaba "detrás de los casos y

\footnotetext{
${ }^{4}$ El LHI es una organización sin fines de lucro con sede en Nueva York cuyo primer presidente fue Richard Weisberg, una de las figuras centrales del momento humanista del movimiento (ver http://docs.law.gwu.edu/facweb/dsolove/Law-Humanities/institute.htm).
} 
reportes judiciales, sirviendo para contrarrestar el rigor formalista de la ley" (Peters, 2005: 444).

Luego de ese momento inicial de los setenta donde se destacan las figuras de J. B. White y Richard Weisberg, centrado fundamentalmente en los textos literarios y priorizando también su diferencia singular frente a la abstracción, las categorías y la sistematización en gran escala de la teoría suele recortarse a los fines heurísticos, un tramo "hermenéutico" que ocupa centralmente los tempranos ochentas y gira de manera casi completa de la literatura, tal como la reconocemos habitualmente, hacia la teoría literaria o la "Teoría" a secas. Este se ha considerado el momento de mayor auge del movimiento, que se centró en el problema de la interpretación en el derecho y acompañó cambios más drásticos en el panorama jurídico. Los objetivos de este período se dirigen en dos direcciones. Por un lado, poner en el centro de la teoría jurídica y sobre todo del derecho constitucional, una dimensión que antes de este momento era considerada periférica, confusa, endeble: la interpretación habría el paso al problema de establecer el sentido del derecho, y enfrentar "la ansiedad a veces ligada al reconocimiento de la inevitabilidad de la interpretación" y la posibilidad ante ello de "encontrar reglas seguras para guiarnos en ese peligroso camino", definir "qué cuenta como 'reglas seguras', si pueden ser 'científicamente' establecidas, y si puede lograrse una independencia de la política $u$ otras perspectivas 'interesadas'"(Levinson y Mailloux, 1989: ix-x). Por otro lado, el "momento hermenéutico" también respondió a necesidades contextuales. Como señalan Levinson y Mailloux, "las invocaciones a la Constitución y las disputas sobre las reglas para su interpretación, son más intensas en tiempos de conflicto político" (1989: 3). Ese clima se vivió durante la victoria de la derecha conservadora de los 80 , con su énfasis en reordenar la justicia para dejar atrás el "activismo judicial" de las décadas pasadas, y en él, la literatura -o más precisamente, la teoría literaria-pareció proveer al derecho de herramientas para "liberarse de la atadura a un texto arcaico y de un grupo de hombres blancos que pretendían custodiarlo (...) y desafiar a las teorías originalistas y textualistas de la interpretación que sostenían las posiciones de una Corte crecientemente reaccionaria" (Peters, 2005: 445).

El arsenal de teoría literaria prometía aquí a los juristas liberar al derecho de su encadenamiento al texto arcaico y al grupo de hombres blancos que lo vigilaban, pero también "amenazaba con desamarrar al derecho de sus bases interpretativas tradicionales", abriendo el problema de la indeterminación que el ala crítica explotaría (Peters, 2005: 445). Dos polémicas pueden considerarse los hitos centrales de este momento: el debate entre R. Dworkin y S. Fish que tuvo como sede la famosa conferencia en la escuela de derecho de Texas de $1981^{5}$, y aquella entre este último y Owen Fiss, que tuviera lugar en San Diego, unos años después ${ }^{6}$.

El "momento narrativo" de los 90 vuelve sobre la mirada humanista que otorgaba un poder de verdad y humanización a la literatura, pero la orienta ahora hacia fines políticos. Impulsado inicialmente por la teoría feminista y la teoría crítica de la raza, fue influido y sostenido también por formaciones institucionales emergentes en esos momentos "que unían la reivindicación psicoterapéutica de una capacidad 'curativa' o 'restaurativa' de las narraciones personales con la reivindicación política del poder transformativo de las narraciones de los oprimidos, como los estudios del 'testimonio', del 'trauma', de la 'memoria' y el establecimiento de comisiones de la verdad donde las víctimas narraban sus historias" (Peters, 2005: 447). Este nuevo modo narrativo de relación del derecho y la literatura se afirma

\footnotetext{
${ }^{5}$ Ambas intervenciones fueron publicadas por primera vez en el volumen de Critical Inquiry editado por W. J. Mitchell (1982).

6 Una reconstrucción de este debate en: Fish, 1984.
} 
también como una reacción ante los exceso de la 'teoría', apareciendo como una perspectiva casi "autoexplicativa" a diferencia de términos como "hermenéutica", "deconstrucción" o "textualidad": "el derecho como narración imagina al derecho como literatura, donde la literatura se presenta como inmediatamente accesible, sin mediación de la teoría" (Binder y Weisberg, 2000: 204) .

Sobre el filo los 90, y con más fuerza durante el comienzo del nuevo milenio, puede situarse la cuarta etapa del movimiento ligada a los "estudios culturales", que volvió a aplanar la diferencia de la literatura en relación a otros discursos, así como a diluir la carga normativa del derecho y a sumergir a ambos en el campo más amplio de la cultura. Es en este momento que el "movimiento derecho y literatura" se amplía hacia denominaciones más inclusivas que ya se sugerían en el título de la publicación de Yale de 1988 (Yale Journal of Law\&theHumanities). La ampliación se profundizaría diez años después, con la primera reunión del grupo de trabajo en "Derecho, cultura y humanidades" en Georgetown (1998), que sería a partir de allí uno de los espacios de sociabilidad clave del remanente del movimiento "derecho y literatura".

Los "estudios culturales" resultan un área heterogénea de difícil definición.En este punto es útil la reconstrucción reciente de Nussbaum porque destaca tanto una lectura a su mejor luz como las limitaciones de este tipo de estudios. Éstos se centran, según Nussbaum, de "maneras más obvias en las contribuciones históricas de las obras literarias al derecho: éstas representan situaciones jurídicas de formas que muestran el funcionamiento de la ley en tiempos y espacios específicos. La otra cara de esta moneda es que los textos literarios pueden ser mejor entendidos si se los estudia en conexión con su contexto jurídico. El movimiento dentro de los estudios literarios 'New Historicism' ha iluminado textos literarios canónicos atendiendo a aspectos del contexto histórico: médicos, legales o culturales" (2013: 11). Dos de los grandes impulsores de los "estudios culturales del derecho" han sido A. Sarat y P. Kahn. Kahn pretendía con este tipo de estudios mover al derecho desde una "teología" hacia una forma de "estudios de religión", desde la prédica y la fe en sí mismo hacia una forma de observación más externa, que "deje de lado el proyecto de reforma, no porque esté satisfecho con las cosas tal como están, sino porque quiere comprender mejor quiénes y qué somos" (Kahn 2001: 46). Sarat, por su parte, pretendía correr el eje literario del movimiento: "las obras literarias no son ya el objeto primario de análisis sino una fuente para entender un problema histórico o cultural más amplio" (Sarat, 2017: 63).

\section{La expansión de las fronteras}

A fines de la década del noventa y con una creciente presencia institucional luego del cambio de milenio, el movimiento "derecho y literatura" se expande más allá de las fronteras norteamericanas y esta expansión trae nuevas preguntas y formas de pensar la relación. En la tercera edición ampliada de Law and Literature, R. Posner señalaba que desde 1998 el movimiento "derecho y literatura" no sólo había crecido en el ámbito interno norteamericano, sino que había migrado hacia Europa: "a los

\footnotetext{
${ }^{7}$ El texto clásico de este período es Law'sStories,compilado por dos de sus figuras salientes: P. Brooks y P. Gewirtz (1996).
} 
países escandinavos en $2005^{8}$, Francia en $2006^{9}$, Inglaterra en $2007^{10}$, Italia ${ }^{11}$ y Portugal en 2008" (2009: xiii). A ello se podría agregar la expansión hacia Australia ${ }^{12}$, España ${ }^{13}$, Latinoamérica ${ }^{14}$ y la creación de una red Europea de intercambio ${ }^{15}$.

En uno de los escasos trabajos comparativos de las diferentes tradiciones del "derecho y la literatura", G. Olson (2010) plantea la necesidad de "desamericanizar" el movimiento repensando tres asunciones ampliamente difundidas en la tradición norteamericana que no se adecúan con facilidad al resto de las culturas jurídicas: el anclaje en el sistema adversarial, la ubicuidad del debate sobre la interpretación constitucional y los modos de la práctica jurídica del commonlaw. Una pregunta semejante aparece en la revisión del panorama europeo de $\mathrm{H}$. Porsdam: "¿qué sucede cuando los textos que se discuten no conforman el canon angloamericano sino que son escandinavos, españoles, holandeses o polacos? ¿qué sucede si estos textos interactúan no con el commonlawsino con tradiciones del civil law? (2009: 174). Una nota particular que presenta la expansión a través de las fronteras del movimiento es que a pesar de haberse iniciado en el momento de auge de importación teórica en su lugar de origen, en el viaje transfronterizo viró el rumbo hacia una preocupación más cercana a los polos que enfatizaban la diferencia en el movimiento, revitalizando momentos que podían considerarse superados. Así, tanto en el movimiento europeo como en el ámbito latinoamericano, las miradas suelen centrarse más que en la teoría, en las obras literarias; y en lecturas que buscan puntos localizados de diálogo con temas y problemas jurídicos más que en la generación de marcos omniabarcativos de amplia escala ${ }^{16}$. En este sentido, Simonin plantea en un trabajo reciente del ámbito francófono: " ¿Y si uno de los futuros posibles del movimiento (...) residiera en un gran salto hacia atrás, en un retorno a los textos literarios? (2015: 72).

\footnotetext{
${ }^{8}$ Una reseña sobre los proyectos y las principales figuras del "derecho y la literatura" nórdicos en: Porsdam, 2009.

${ }^{9}$ En el ámbito francófono suelen mencionarse dos hitos tempranos durante los primeros años de los 80 s: la creación en 1982 de un curso sobre Droit et Littérature en Université Paris Diderot; la organización en 1984 de un congreso sobre el tema (Trindade y Gubert, 2009:180). Pero es recién a fines de los 90s que se publica la primera antología (Maluire, 1997).

${ }^{10}$ En este caso, los cruces pueden datarse mucho antes, en el seno de los estudios críticos del derecho británicos durante la década del 80 (Olson, 2010:346).

11 Una revisión de los estudios en derecho y literatura italianos en Trindade y Gubert(2009) y Mittica(2015)

12 En abril de 1990 se organizó la primera conferencia sobre "derecho y literatura" en Australia y se consolidó la Asociación Australiana en Derecho y Literatura. Uno de sus miembros fundadores, J. Neville Turner, compiló unos años después el primer volumen australiano en "derecho y literatura": TheHappyCouple: Law and Literature (1994). En noviembre de 2011 se conformó una red más amplia para la región de Australasia (ver http://lawlithum.org/about/).

13 Una revisión de la recepción en España del movimiento, en: Arsuaga, 2009.

14 Una serie de trabajos sobre el derecho y la literatura firmados por autores latinoamericanos se encuentra en el dossier coordinado por el ecuatoriano D. Falconi (2016). El caso brasilero es quizás el más desarrollado a nivel institucional de la región: desde 2014 cuenta con una red nacional de derecho y literatura que incluye un programa de TV, una publicación académica especializada y un "Coloquio internacional de derecho y literatura" anual (conf. http://rdl.org.br/). Sobre el "derecho y la literatura" en Brasil: Trindade y Bernsts, 2017. Un punteo de las figuras y trabajos dedicados al derecho y la literatura en Argentina, en: Roggero, 2016.

${ }^{15} \mathrm{La}$ red europea de derecho y literatura fue lanzada a instancias de Greta Olson y Jeanne Gaaker. La publicación más reciente sobre esta tradición es Porsdam y Elholm (2012).

${ }^{16}$ En la introducción a un volumen reciente que agrupa trabajos del ámbito francófono, Garapon y Salas señalan que la perspectiva que nuclea a estos trabajos es una que se concentra "en la manera en que la ficción literaria reflexiona sobre el mundo jurídico", frente a "el derecho como literatura, (...) punto de vista dominante en Estados Unidos" (2015:14)
} 


\section{La migración hacia los estudios literarios: Literatura y Derechos Humanos}

Una de las características particulares del movimiento derecho y literatura es su migración desde la sede jurídica en la que se había desarrollado durante más de tres décadas, hacia el terreno de los estudios literarios bajo la denominación "Literatura y Derechos Humanos". Este nuevo desarrollo del movimiento, por otra parte, señala tanto una nueva oscilación en la carrera del movimiento hacia formas de pensar la relación que parten de la diferencia de la literatura, como también un retorno a los estadios iniciales que se creían superados y caídos en el olvido luego de los períodos de ascendencia teórica de los 80 y los primeros años del 2000.

Esta especie de retorno o revisión de las fuentes humanísticas del movimiento, aún en transcurso en la actualidad, también puede ligarse a la crisis de las humanidades y el replanteamiento de sus protocolos de lectura. Gran parte de la bibliografía que rastrea la emergencia del campo "literatura y derechos humanos" lo sitúa luego de los sucesos del 11/9 de 2001 en el Norte Global (Goldberg y Moore, 2012; Brooks, 2014). La fragilidad de los derechos humanos en el contexto precario del neoliberalismo, el auge de los discursos de la seguridad nacional y sus culturas del miedo han sido considerados los factores que explican parcialmente "la urgencia por encontrar en las humanidades, especialmente en la literatura, el antídoto a la crueldad, las atrocidades y las violaciones de los derecho humanos" (McClennen 2013: 180). A ello se sumó también la necesidad de "defender a las humanidades" en un contexto de precarización y repliegue de la educación humanística en pos de lo que Nussbaum (2010) llamó "el modelo de educación económico", y en este punto, el giro hacia los derechos humanos en las humanidades era también, de manera correlativa a lo que había sucedido en los inicios del movimiento en el campo del derecho, una búsqueda de articulación positiva del valor de la literatura y las humanidades, sólo que ahora esos valores se buscaban del lado y en asociación con el derecho.

Aún en un terreno en proceso de formación, dentro de la "literatura y los derechos humanos" pueden rastrearse una serie de líneas de investigación interrelacionadas que pretenden renovar tanto las miradas sobre los derechos humanos como también transformar internamente el ámbito de los estudios literarios, buscando modos de dar una respuesta a la relevancia de la literatura y articular un vocabulario específico para dar cuenta de ese valor. Dentro de los aún escasos intentos de dotar de forma al campo se han señalado dos líneas que se han transitado en la vinculación entre los derechos humanos y la literatura: una "conecta históricamente términos clave de derechos humanos a innovaciones estéticas específicas" o "lee textos literarios en cuanto representan y vuelven inteligibles las filosofías y las prácticas de derechos humanos en distintos contextos culturales"; otra, examina la relación entre "luchas por la justicia y su expresión a través de formas literarias" (McClennen y Moore, 2016: 9; Goldberg y Moore, 2012: 10). Por su parte, K. Bystrom (2012) agrega una tercera, aún incipiente, centrada en el "acto de lectura" más que en los textos o las técnicas de lectura que dicta la teoría, que considera capaz de abrir los nuevos rumbos para el movimiento.

\section{A modo de cierre.}

El movimiento "derecho y la literatura" es un espacio interdisciplinario que surgió avanzada la segunda mitad del siglo XX, originalmente en la academia norteamericana, dedicado a explorar las relaciones entre ambos términos. Uno de sus impulsos iniciales, que se mantuvo a la largo de su trayectoria, era enfrentar el formalismo y tecnicismo de la práctica y la educación jurídica. Desde la década de los 70 , su trayectoria fue girando hacia distintos intereses, temas y problemas; e incluso, 
en los primeros años del milenio, no sólo se expandió a través de las fronteras sino que también migró de su sede jurídica original hacia los estudios literarios. Este trabajo se propuso revisar sus orígenes, repasar sus hitos, autores, instituciones y contextos fundamentales, y ofreció para ello una periodización distinguible en su trayectoria que incluye un panorama de su expansión transfronteriza y de sus nuevas dimensiones en el ámbito de los estudios literarios.

Bibliografía

AMAYA, A. (2013), "Derecho y Literatura".En: AULLÓN DE HARO, P. (ed.), Metodologías comparatistas y literatura comparada, Madrid, Dykinson, pp. 173182.

ANKER, E. Y MEYLER, B. (2017),New directions in Law and Literature, Oxford University Press, New York.

ARSUAGA, T. (2009) "Derecho y literatura. Origen, tesis principales y recepción en España", Workingpaper IE LawSchool, AJ, pp. 8-157.

BALKIN, J. (1996), "Interdisciplinarity as colonization", Washington \& Lee Law Review, 53,pp. 949-970.

BINDER, G. Y WEISBERG, R. (2000), Literary Criticism of Law,Princeton U. Press, New Jersey.

BYSTROM, K.(2012), "Literature and Human Rights".En CUSHMAN, T. (ed.) Handbook of Human Rights,New York, Routledge, pp.637-646.

BOURDIEU, P. (1995) Las reglas del arte, Anagrama, Barcelona.

BROOKS, P. y GEWIRTZ, P. (eds.) (1996), Law's Stories, Yale U. Press, New Haven.

BROOKS, P.y JEWETT, H. (eds.) (2014), The Humanities and the Public Life, Fordham U. Press, New York.

CARDOZO, B. (1925), "Law and Literature", Yale Review,14,pp. 699-718.

FALCONÍ TRÁVEZ, D. (coord.) (2016), "Dossier Derecho y Literatura en América Latina", luris Dictio, $\mathrm{n}^{\circ} 18$.

FISH, S. (1984), "Fish v. Fiss", Stanford L. Rev., v. 36, n.6, pp. 1325-1347.

GARAPON, A. y SALAS, D. (eds.) (2015), Imaginar la ley. El derecho en la literatura, Jusbaires, Buenos Aires.

GOLDBERG, E. y MOORE, A. (eds.) (2012), Theoretical perspectives on Human Rights and Literature, Routledge, New York.

KAHN, P.(2001), El análisis cultural del derecho,Gedisa, Barcelona.

MCCLENNEN, S. (2013), "Theoretical Perspectives on Human Rights and Literature (review)", College Lit.,V. 40, N. 3, pp. 180-182.

MCCLENNEN, S. y MOORE,A. (eds.) (2016), Routledge Companion to Literature and Human Rights, Routledge, New York.

MALAUIRE, P. (1997),Droit et Littérature. Une Anthologie,Cujas,Paris.

MITCHELL, W.J.T (ed.) (1982), "The Politics of Interpretation", Critical Inquiry, V.9, N.1.

MITTICA, P. (2009), "Diritto e letteratura in Italia. Statodell'arte e riflessionisul método", Materiali per una storiadella cultura giuridica, n. 1, pp. 273-299.

NUSSBAUM, M. (1997), Justicia Poética, Andrés Bello, Santiago de Chile.

NUSSBAUM, M. (2006), El conocimiento del amor. Ensayos sobre filosofía y literatura, A. Machado, Madrid.

NUSSBAUM, M. (2010), Sin fines de lucro. Por qué la democracia necesita de las humanidades, Katz, Buenos Aires.

NUSSBAUM, M. y LACROIX, A. (2013), Subversion and Sympathy: Gender, Law, and the British Novel, Oxford U. Press, New York.

LEVINSON, S. y MAILLOUX, S. (eds.) (1989),Interpreting Law and Literature, Northwestern University Press, Evanston. 
OLSON, G. (2010), "De-Americanizing Law and Literature Narratives: Opening Up the Story", Law \& Literature, V. 22, Iss. 2, pp. 338-364.

PORSDAM, H. (2009), From Civil to Human Rights. Dialogues on Law and Humanities in the United States and Europe,Edward Elgar, Cheltenham.

PORSDAM, H. y ELHOLM, T. (2012),Dialogues on Justice: European Perspectives on Law and Humanities, De Gruyter, Berlín.

POSNER, R. (2009), Law and Literature ( $3^{\circ}$ ed.), Harvard U. Press, Cambridge.

ROGGERO, J. (2016), "Hay derecho y literatura en Argentina", Anamorphosis, V.2, N.2, pp.269-292.

SÁENZ, M.J. (2017), "Derechos humanos y literatura: un espacio emergente de encuentro entre el derecho y la literatura", Anamorphosis, V.3,N.1,pp.5-24.

SARAT, A. (1998) "Traditions and Trajectories in Law and Humanities", Yale Journal of Law \& Humanities, V. 10, Iss. 2, pp. 401-407.

SARAT, A. (2017), "From Charisma to Routinization and Beyond: Speculations on the Future of the Study of Law and Literature". En: ANKER, E.S. y MEYLER, B.,New Directions in Law and Literature, Oxford University Press, New York, pp. 59-66.

SIMONIN, A. (2015), "MaketheUnorthodoxOrthodox: J. H. Wigmore y el nacimiento del interés del derecho por la literatura". En: GARAPON y SALAS (eds.) (2015), Imaginar la ley. El derecho en la literatura, Buenos Aires, Jusbaires, pp. 33-76.

THOMAS, B. (1991), "The Law and Literature Revival", Critical Inquiry, V. 17, N.3, pp.510-539.

TRINDADE, A. Y GUBERT, R. (2009), "Derecho y literatura. Acercamientos y perspectivas para repensar el derecho", Revista del Instituto "Ambrosio L. Gioja", Año III, N. 4, pp. 164-213.

TRINDADE, A. y BERNSTS, L. (2017), "The Study of Law and Literature in Brazil: development, evolution and expansion", Anamorphosis, V.3,N.1, pp.225-57.

WEISBERG, R. (1989), "The Law and Literature Enterprise", Yale Journal of Law \& Humanities, V.1, Iss. 1, pp. 1-67.

WIGMORE, J. (1908) "A List of Legal Novels", Illinois Law Rev, pp. 574-593.

WHITE, J.B. (1973), The Legal Imagination, Chicago U. Press, Chicago.

YOSHINO, K. (2006), "La ciudad y el poeta",Rev. Jur. de la Universidad de Palermo, Año 7, N. 2, pp. 5-61. 